\title{
THERE ARE NO DEVIATIONS FOR THE ERGODIC AVERAGES OF THE GIULIETTI-LIVERANI HOROCYCLE FLOWS ON THE TWO-TORUS
}

\author{
VIVIANE BALADI
}

\begin{abstract}
We show that the ergodic integrals for the horocycle flow on the two-torus associated by Giulietti and Liverani to an Anosov diffeomorphism either grow linearly or are bounded, in other words there are no deviations. For this, we use topological invariance of the Artin-Mazur zeta function to exclude resonances outside of the open unit disc. Transfer operators acting on suitable spaces of anisotropic distributions and their Ruelle determinants are the key tools in the proof. As a bonus, we show that for any $C^{\infty}$ Anosov diffeomorphism $F$ on the two-torus, the correlations for the measure of maximal entropy and $C^{\infty}$ observables decay with a rate strictly smaller than $e^{-h_{t o p}(F)}$. We compare our results with very recent related work of Forni.
\end{abstract}

\section{INTRODUCTION}

1.1. The results of Giulietti-Liverani. In a pioneering work, Giulietti and Liverani [7 introduced a "horocycle flow" on the torus, renormalizable by a given Anosov diffeomorphism: Fix $r>1$ and let $F: \mathbb{T}^{2} \rightarrow \mathbb{T}^{2}$ be a $C^{r}$ Anosov diffeomorphism on the two-torus. By Franks-Newhouse the stable bundle $E^{s}$ of $F$ (see [13, p. 805]) is orientable. Fixing an orientation $E_{+}^{s}$ of $E^{s}$, Giulietti and Liverani assume that $D F$ preserves this orientation and they introduce the flow $h^{t}=h_{s}^{t}$ on $\mathbb{T}^{2}$ obtained by solving $\partial_{t} h^{t}(f)=X(f)$, where $X(x)=v_{1,+}^{s}(x)$ is the unique vector of $E_{+}^{s}(x)$ of unit norm. We call $h^{t}$ the (uni speed) Giulietti-Liverani (stable horocycle) flow (of $F$ ). (See Appendix $\mathrm{A}$ for basic facts about such flows.)

For any continuous function $f: \mathbb{T}^{2} \rightarrow \mathbb{C}$, any $T>0$, and any $x \in \mathbb{T}^{2}$, define the horocycle integral $H_{x, T}(f)=\int_{0}^{T} f\left(h^{t}(x)\right) d t$.

By unique ergodicity, we have for any such $x$ and $f$

$$
\lim _{T \rightarrow \infty} \frac{H_{x, T}(f)}{T}=\mu^{s}(f):=\int_{\mathbb{T}^{2}} f(x) d \mu^{s},
$$

Date: December 16, 2020.

Most of this work was done while preparing and delivering a minicourse on Anisotropic spaces and applications to hyperbolic and parabolic dynamical systems (Oberwolfach, June 2019). VB is very grateful to MFO for offering this possibility. Thanks to Romain Dujardin, Giovanni Forni, Sébastien Gouëzel, and Carlangelo Liverani for useful discussions and to Paolo Giulietti for pointing out typos. Thanks also to the anonymous referee and the editor for comments which helped improve the presentation. VB's research is supported by the European Research Council (ERC) under the European Union's Horizon 2020 research and innovation programme (grant agreement No 787304).

${ }^{1}$ Giulietti and Liverani actually consider more general $C^{r}$ time parametrizations but this is immaterial for the purposes of this work, as the resonances defined below are invariant under such time-changes since the transfer operator is modified by a $C^{r}$ coboundary [ 7.5 ]. 
where $\mu^{s}$ is the unique invariant probability measure of the flow $h^{t}$.

Let $h_{\text {top }}=h_{\text {top }}(F)>0$ be the topological entropy of $F$. Giulietti and Liverani 7 find $r_{0}>1$ such that, if $r \geq r_{0}$ there exists a bounded linear operator $\widetilde{\mathcal{L}}$ associated with $F$ (see (2.3) below) acting on a Banach space $\widetilde{\mathcal{B}}_{G L}$ of distributions in a subset of the unit tangent bundle of $\mathbb{T}^{2}$ such that the following holds: The spectral radius of $\widetilde{\mathcal{L}}$ on $\widetilde{\mathcal{B}}_{G L}$ is $e^{h_{t o p}}$ and the essential spectral radius of $\widetilde{\mathcal{L}}$ is strictly smaller than $\tilde{\rho}_{G L}=\tilde{\rho}_{G L}(r)<1$. In addition, $\tilde{\rho}_{0}:=e^{h_{t o p}}$ is a simple eigenvalue and the only maximal eigenvalue. Restricting for simplicity to the case where $\widetilde{\mathcal{L}}$ does not have any eigenvalue of modulus one and, in addition, all of its eigenvalues of modulus larger than $\tilde{\rho}_{G L}$ have trivial Jordan blocks (otherwise, additional factors $\log T^{q}$ with $q \geq 1$ can appear in the expansion (1.2) below), we let $\left\{\tilde{\rho}_{j}\right\}_{j=1}^{N_{G L}}$ be the $N_{G L} \geq 0$ eigenvalues of $\widetilde{\mathcal{L}}$ of modulus $\tilde{\rho}_{G L} \leq\left|\tilde{\rho}_{j}\right|<e^{h_{t o p}}$ (ordered by decreasing modulus and repeated with multiplicity), and we let $\mathcal{O}_{j} \in \widetilde{\mathcal{B}}_{G L}^{*}$ be the associated eigenvectors of the dual operator $\tilde{\mathcal{L}}^{*}$. We call $\left\{\tilde{\rho}_{j}\right\}_{j=0}^{N_{G L}}$ the resonance $\boldsymbol{Z}_{\text {of }} \widetilde{\mathcal{L}}$. We call the $N_{1, G L} \leq N_{G L}$ resonances such that $1 \leq\left|\tilde{\rho}_{j}\right|<e^{h_{t o p}}$ its deviation resonances. By [9] (see also [7, Lemma 2.11]), the maximal eigenvector $\widetilde{\mathcal{L}}^{*} \tilde{\mu}^{s}=e^{h_{\text {top }}} \tilde{\mu}^{s}$ satisfies $\pi^{*}\left(\tilde{\mu}^{s}\right)=\mu^{s}$ (this fixes a normalisation of $\tilde{\mu}^{s}$ ), where $\pi$ denotes the projection from the unit tangent bundle of $\mathbb{T}^{2}$ to $\mathbb{T}^{2}$.

The first main result of Giulietti-Liverani [7, Theorem 2.8] gives the following expansion for the horocycle integral $H_{x, T}(f)$ : For any $\delta \in\left(0,-\log \tilde{\rho}_{G L}\right)$, there exists a constant $C<\infty$ such that, for any $T>0$, and any $x \in \mathbb{T}^{2}$ there exist real numbers $\left\{C_{j}(x, T)\right\}_{j=0}^{N_{G L}}$ with $\sup _{x, T, j}\left|C_{j}(x, T)\right| \leq C$, such that for any $C^{r}$ function $f: \mathbb{T}^{2} \rightarrow \mathbb{C}$, we have

$$
H_{x, T}(f)=T \cdot C_{0}(x, T) \cdot \mu^{s}(f)+\sum_{j=1}^{N_{G L}} T^{\theta_{j}} \cdot C_{j}(x, T) \cdot \mathcal{O}_{j}(f \circ \pi)+\mathcal{R}_{x, T}(f),
$$

where $\theta_{j}=\frac{\log \left|\tilde{\rho}_{j}\right|}{h_{\text {top }}} \in(-\infty, 1)$ and, for any $T>0$,

$$
\sup _{x}\left|\mathcal{R}_{x, T}(f)\right| \leq C\left(T^{\theta_{\text {min }}}\|f\|_{C^{r}}+\sup |f|\right), \text { where } \theta_{\text {min }}=\frac{\log \tilde{\rho}_{G L}+\delta}{h_{\text {top }}}<0 .
$$

Decomposing $f=\mu^{s}(f)+f-\mu^{s}(f)$, we get

$$
T \cdot C_{0}(x, T)=H_{x, T}(1)=\int_{0}^{T} 1 d t=T,
$$

up to replacing $\mathcal{O}_{j}(f \circ \pi)$ by $\mathcal{O}_{j}\left(\left(f-\mu^{s}(f)\right) \circ \pi\right)$ in the right-hand-side of (1.2).

We refer to the introduction of 7 for the motivation arising from the well-known works of Forni and Flaminio giving expansions of ergodic averages along horocycles of (e.g.) geodesic flows on compact surfaces of constant negative curvature in terms of eigenvalues of the Laplacian and of horocycle-flow invariant distributions (see e.g. [5). Some of the results of Flaminio and Forni have been extended to variable negative curvature geodesic flows [1] by adapting the ideas in [7.

\footnotetext{
${ }^{2}$ We shall see after (2.6) below that they do not depend on the Banach space.

${ }^{3}$ Giulietti-Liverani 7 limit the expansion to $\left|\tilde{\rho}_{j}\right| \geq 1$, i.e. $\theta_{j} \geq 0$, bounding the error term by $C\|f\|_{C^{r}}$. They write $\ell_{j}(x, T, f)$ instead of $C_{j}(x, T) \cdot \mathcal{O}_{j}(f \circ \pi)$. Their proof gives the slightly more precise result 1.2 .
} 
Remark 1.1 (Bound for $r_{0}$ ). An upper bound for $r_{0}$ can be read off from the bound for $\tilde{\rho}_{G L}(r)$ in [9], noting that the limit is $r$ and not $r-1$ in the case $\iota=1$ there, and taking care of the fact that one of the regularity exponents is an integer. In [7, the authors mention that choosing $r_{0} \geq 1$ large enough such that

$$
e^{h_{\text {top }}(F)} / \lambda^{r_{0} / 2}<1
$$

suffices (where $1<\lambda<\min \left\{\lambda_{u}, 1 / \lambda_{s}\right\}$, see (2.5), and $\lambda_{u}, \lambda_{s}$ are defined in the

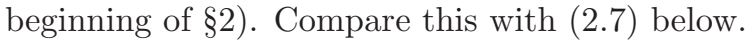

1.2. Absence of deviation resonances and two consequences. The main technical result of the present work, Theorem 2.1, is that, if $r \geq r_{1}$ is large enough $\left(r_{1}\right.$ depends on the expansion and contraction factors of $F$, see (2.7) and (2.8) ) then $\left|\tilde{\rho}_{j}\right|<1$ for all $j=1, \ldots, N_{G L}$. (In othel words, the transfer operator $\widetilde{\mathcal{L}}$ does not have deviation resonances.) As a consequence (Corollary 2.3), if $r \geq \max \left\{r_{0}, r_{1}\right\}$, the exponents $\theta_{j}$ in the expansion (1.2) all satisfy $\theta_{j}<0$. In particular (even in the presence of Jordan blocks), there exists $C^{\prime}<\infty$ and $\theta_{\text {min }}^{\prime}<0$ such that

$$
\sup _{x}\left|H_{x, T}(f)-T \cdot \mu^{s}(f)\right| \leq C^{\prime}\left(T^{\theta_{\text {min }}^{\prime}}\|f\|_{C^{r}}+\sup |f|\right), \forall T>0 .
$$

That is, there are no deviations to the convergence of horocycle ergodic integrals.

We next mention a consequence of Theorem 2.1 about the "cohomological equation." Since $h^{t}(x)$ is minimal, Gottschalk-Hedlund's theorem (see 14 for a recent account) implies, for any continuous $f$, that $\sup _{x, T}\left|H_{x, T}(f)\right|<\infty$ if and only if $f$ is a continuous coboundary, i.e. there exists a continuous function $\bar{f}$ such that for all $x$ and all $T>0$, the following cohomological equation holds:

$$
\bar{f}(x)-\bar{f} \circ h^{T}(x)=\int_{0}^{T} f \circ h^{t}(x) d t .
$$

The expansion (1.4) from Corollary 2.3 thus implies the following dichotomy for the Giulietti-Liverani flow of a $C^{r}$ Anosov diffeomorphism with $r \geq \max \left\{r_{0}, r_{1}\right\}$ : If $f \in C^{r}$ then either $\mu^{s}(f) \neq 0$ (and $H_{x, T}(f)$ grows linearly) or $f$ is a continuous coboundary.

Another immediate consequence of Theorem 2.1 about the absence of deviation resonances for $\widetilde{\mathcal{L}}$ is Corollary 2.5. For any $C^{\infty}$ Anosov diffeomorphism $F$ on the two-torus, the correlations for the unique measure of maximal entropy $\mu_{\text {top }}$ and $C^{\infty}$ observables decay with rate strictly smaller than $e^{-h_{t o p}(F)}$. (In fact Corollary 2.5 only requires $C^{r}$ for large enough $r$.)

Giovanni Forni [6] obtained (independently and simultaneously) related result:5, in a more general setting: For any $C^{r}(r>1)$ pseudo Anosov diffeomorphism on a compact surface, the correlation spectrum of the Margulis measure is determined by the action of the diffeomorphism on the first cohomology, up to a power law error term (his results do not imply Corollary 2.5 even when the diffeomorphism is Anosov and $r$ is large, see Remark 2.6).

We end this introduction by mentioning that if $F$ is a $C^{r}$ Anosov diffeomorphism on a compact connected $C^{\infty}$ manifold $M$ of dimension $d \geq 2$, and the dimension $d_{s}$ of its stable bundle is equal to one (this is the situation when a horocycle flow

\footnotetext{
4 "Men han har jo ikke noget paa," sagde et lille Barn.

${ }^{5}$ We focus on the analogue of Corollary 2.5 but Forni's main result is an analogue of Corollary 2.3 about equidistribution of stable or unstable curves.
} 
of Giulietti-Liverani type can be constructed), then $M$ is homeomorphic (and thus diffeomorphic) to the torus $\mathbb{T}^{d}$, and $F$ is topologically conjugated to a linear toral automorphism $A$ of $\mathbb{T}^{d}$ (see 13] for a recent account of this result of Franks and Newhouse). See Remark 3.1 about the limits of our approach in dimensions $d \geq 3$.

Remark 1.2. After this paper was accepted for publication, Jérôme Carrand [4, following a suggestion of Selim Ghazouani, used Denjoy-Koksma to give a very short proof of a slightly weaker result (applying to a slightly larger class of flows): the ergodic integrals of a zero-average observable grow at most logarithmically.

\section{Statement of the MAin theorem AND its TWO COROLlaries}

Fix $r>1$ and let $F: \mathbb{T}^{2} \rightarrow \mathbb{T}^{2}$ be a $C^{r}$ Anosov diffeomorphism on the two-torus. This means that there exist $C<\infty$ and $\lambda_{u}>1, \lambda_{s}<1$ such that the tangent space splits as $T \mathbb{T}^{2}=E^{s} \oplus E^{u}$, with $E^{s}$ and $E^{u}$ preserved by $D F$, such that, for all $x$ 国

$\left\|D F_{x}^{n} v\right\| \leq C \lambda_{s}^{n}\|v\|, \forall v \in E^{s}(x),\left\|D F_{x}^{-n} v\right\| \leq C \lambda_{u}^{-n}\|v\|, \forall v \in E^{u}(x), \forall n \geq 1$.

By Franks-Newhouse (see e.g. [13), since $d_{s}=1$, the Anosov diffeomorphism $F$ is topologically conjugated to a hyperbolic linear toral automorphism. In particular, $F$ is topologically mixing. In fact, $d_{s}=1$ also implies that $E^{s}$ (see [13, p. $805])$ is orientable. Fixing an orientation of $E^{s}$, we assume that $D F$ preserves this orientation.

2.1. The Artin-Mazur zeta function of $F$. Let $A=A_{F} \in G l_{2}(\mathbb{Z})$ be the hyperbolic matrix topologically conjugated to $F$ (i.e. the unique linear map in the homotopy class of $F$ ). The eigenvalue 7 of $A$ are

$$
\sigma e^{h_{t o p}(F)} \text { and } e^{-h_{\text {top }}(F)}<1 \text {, with } \sigma:=\sigma_{A}=\operatorname{det} A \in\{ \pm 1\} .
$$

(The contracting eigenvalue is positive by our orientation preserving assumption.) Put $\lambda_{A}=e^{h_{t o p}(F)}$. An easy computation gives that the unweighted (Artin-Mazur) zeta function of $A$ is just

$$
\zeta_{A}(z)=\exp \sum_{n=1}^{\infty} \frac{z^{n}}{n} \# \operatorname{Fix} A^{n}= \begin{cases}\frac{(1-z)^{2}}{\left(1-\lambda_{A} z\right)\left(1-z / \lambda_{A}\right)} & \text { if } \sigma=+1 \\ \frac{1-z^{2}}{\left(1-\lambda_{A} z\right)\left(1+z / \lambda_{A}\right)} & \text { if } \sigma=-1 .\end{cases}
$$

(Use 8 that \#Fix $A^{n}=\left|\operatorname{det}\left(1-A^{n}\right)\right|=\left|1-\left(\sigma \lambda_{A}\right)^{n}\right| \cdot\left(1-\lambda_{A}^{-n}\right)$ so that \#Fix $A^{n}=$ $-\left(-\lambda_{A}\right)^{n}-\lambda_{A}^{-n}=\lambda_{A}^{n}+(-1)^{n} \lambda_{A}^{-n}$ if $\sigma=-1$ and $n$ is odd, while \#Fix $A^{n}=$ $-2+\lambda_{A}^{n}+\lambda_{A}^{-n}$ otherwise, and handle separately even and odd $n$ in this case, using that $\exp \sum_{j=1}^{\infty} 2 \cdot z^{2 j} / 2 j=\left(1-z^{2}\right)$.)

2.2. The extended dynamics $\tilde{F}$ and the transfer operator $\widetilde{\mathcal{L}}$. Identifying $T_{x} \mathbb{T}^{2}$ with $\mathbb{R}^{2}$ for any $x \in \mathbb{T}^{2}$, we let $x \mapsto \mathcal{C}^{s}(x) \subset T_{x} \mathbb{T}^{2}$ be a smooth stable cone field for $F$, that is, each $\mathcal{C}^{s}(x)$ is a strict subset of $\mathbb{R}^{2}$ of nonempty interior, with $\xi \cdot \mathcal{C}^{s}(x) \subset \mathcal{C}^{s}(x)$ for all $\xi \in \mathbb{R}$, and such that

$$
\overline{D F^{-1}\left(\mathcal{C}^{s}(x)\right)} \subset \mathcal{C}^{s}\left(F^{-1}(x)\right) \cup\{0\}, \forall x \in \mathbb{T}^{2},
$$

\footnotetext{
${ }^{6}\|\cdot\|$ denotes the norm on $T_{x} \mathbb{T}^{2}$ induced by the Riemann metric on $\mathbb{T}^{2}$.

${ }^{7}$ We can again reduce to the case $\sigma_{A}=1$ by considering $F^{2}$, but we find it instructive to write the zeta function in the general case.

${ }^{8}$ See e.g. the proof of [10, Lemma 18.6.2].
} 
and there exists $\nu_{s}<1$ such that

$$
\left\|D F_{x}^{-1}(v)\right\| \geq \nu_{s}^{-1}\|v\|, \forall x \in \mathbb{T}^{2}, \forall v \in C(F(x)) .
$$

We consider the following compact subspace of the unit tangent bundle of $\mathbb{T}^{2}$ :

$$
\Omega^{s}=\left\{(x, v) \in \mathbb{T}^{2} \times \mathbb{R}^{2} \mid\|v\|=1, \quad v \in \overline{\mathcal{C}^{s}(x)}\right\} .
$$

Orientability of $E^{s}$ gives a decomposition $\Omega^{s}=\Omega_{+}^{s} \cup \Omega_{-}^{s}$. Since $D F$ preserves the orientation of $E^{s}$, the $C^{r-1}$ map defined on $\left\{(x, v) \in \Omega_{+}^{s} \mid D F_{x}(v) \in \overline{\mathcal{C}^{s}(x)}\right\}$ by

$$
\tilde{F}(x, v)=\left(F(x), \frac{D_{x} F(v)}{\left\|D_{x} F(v)\right\|}\right)
$$

leaves invariant the set

$$
E_{1,+}^{s}:=\left\{(x, v) \in \Omega_{+}^{s} \mid v \in E^{s}(x)\right\}=\left\{\left(x, v_{1,+}^{s}(x)\right) \mid x \in \mathbb{T}^{2}\right\} .
$$

It is easy to see that $\left(\tilde{F}, E_{1,+}^{s}\right)$ is a topologically mixing Axiom A repellor, with stable dimension one and unstable dimension two, and that the expansion in the new unstable direction is not smaller than $\lambda_{u} / \nu_{s}>\lambda_{u}$. For each $n \geq 1$, the periodic orbits of $F^{n}$ are in bijection with the periodic orbits of $\tilde{F}^{n}$ via the map $x \mapsto\left(x, v_{1,+}^{s}(x)\right)$. Following [7], we set

$$
\tilde{g}(x, v)=\frac{1}{|\operatorname{Det} D F|_{V} \mid \circ \tilde{F}^{-1}(x, v)},
$$

where $V\left(\tilde{F}^{-1}(x, v)\right)=\mathbb{R} \cdot D F_{x}^{-1} v \subset T_{F^{-1}(x)} \mathbb{T}^{2}$ is the line generated by $D F_{x}^{-1} v$, and we consider the weighted transfer operator $\widetilde{\mathcal{L}}$ of $\tilde{F}$ defined by

$$
(\widetilde{\mathcal{L}} \tilde{\varphi})(x, v)=(\tilde{g} \tilde{\varphi}) \circ \tilde{F}^{-1}(x, v) .
$$

(As explained in [7, the operator $\widetilde{\mathcal{L}}$ corresponds to the action of $F$ on one-forms.)

2.3. The essential spectrum of $\widetilde{\mathcal{L}}$ on the Banach space $\widetilde{\mathcal{B}}$. Since $\lambda_{u} / \nu_{s}>\lambda_{u}$, and $\tilde{d}_{s}=1$, if $r<\infty$ the result:9] of [3, Theorem 1.1] (see also [2, Chap. 5] for a pedestrian account) imply that for any $\epsilon>0$, there exists a Banach space $\widetilde{\mathcal{B}}=\widetilde{\mathcal{B}}^{t, s}(\tilde{F})$ of anisotropic distributions on $\Omega_{+}^{s}$ (supported in a neighbourhood $\mathcal{E}$ of $\left.E_{1,+}^{s}\right)$ such that the essential spectral radius of $\widetilde{\mathcal{L}}$ on $\widetilde{\mathcal{B}}$ is strictly 10 smaller than

$$
\begin{aligned}
\tilde{\rho}_{B T}:=\tilde{\rho}_{B T}(r) & =\exp \max _{\substack{s, t \in \mathbb{R} \\
t-(r-1-\epsilon) \leq s<0<t}} \sup _{\tilde{\mu} \in \operatorname{Erg}(\tilde{F})}\left\{h_{\tilde{\mu}}+\max \left\{-t \ell_{\tilde{\mu}}^{u},|s| \ell_{\tilde{\mu}}^{s}\right\}\right\} \\
& =\exp \max _{\substack{s, t \in \mathbb{R} \\
t-(r-1-\epsilon) \leq s<0<t}} \sup _{\mu \in \operatorname{Erg}(F)}\left\{h_{\mu}+\max \left\{-t \ell_{\tilde{\mu}}^{u},|s| \ell_{\mu}^{s}\right\},\right.
\end{aligned}
$$

where $\ell_{\tilde{\mu}}^{u / s}=\int \log \left(\left.\operatorname{det} D F\right|_{E^{u / s}}\right) d \tilde{\mu}$, and $\operatorname{Erg}(\tilde{F})$ is the set of ergodic $\tilde{F}$-invariant probability measures, which is in bijection with $\operatorname{Erg}(F)$, with $h_{\tilde{\mu}}=h_{\mu(\tilde{\mu})}$ and $\ell_{\tilde{\mu}}^{u / s}=\ell_{\mu(\tilde{\mu})}^{u / s}$, where $\ell_{\mu}^{u / s}=\int \log \left(\left.\operatorname{det} D F\right|_{E^{u / s}}\right) d \mu$. (The $\ell^{u}$ are strictly positive and the $\ell^{s}$ strictly negative.)

Note that by [2, Chap. 5] we have $\tilde{\rho}_{B T} \leq \frac{e^{h_{t o p}(F)}}{\lambda^{(r-1) / 2}}$ for any $\lambda$ such that

$$
1<\lambda<\min \left\{\lambda_{u}, 1 / \lambda_{s}\right\}
$$

\footnotetext{
${ }^{9}$ Theorem 2.1 there shows that one does not need to replace $r-1$ by $r-2$.

${ }^{10}$ Take the Banach space $C^{t, s}(\tilde{F}, \mathcal{E})$ in $[3$ Theorem 1.1], for $\epsilon>0$ and $t-(r-1-\epsilon)<s<0<t$.
} 
Since $\tilde{F}$ is mixing, $\tilde{\rho}_{0}:=e^{h_{t o p}}$ is a simple eigenvalue and the only maximal eigenvalue. Set

$$
\tilde{\rho}_{e s s}:=\max \left\{\tilde{\rho}_{G L}, \tilde{\rho}_{B T}\right\} .
$$

The sets $\Sigma_{G L}:=\left\{\tilde{\rho} \in \operatorname{sp}\left(\widetilde{\mathcal{L}}_{\widetilde{\mathcal{B}}_{G L}}\right)|| \tilde{\rho} \mid>\tilde{\rho}_{\text {ess }}\right\}$ and $\Sigma_{B T}:=\left\{\tilde{\rho} \in \operatorname{sp}\left(\left.\widetilde{\mathcal{L}}\right|_{\tilde{\mathcal{B}}}\right)|| \tilde{\rho} \mid>\tilde{\rho}_{\text {ess }}\right\}$ coincide (including multiplicities) by [3, App. A.2]. In addition, the finitely many corresponding generalised eigenvectors lie in $\widetilde{\mathcal{B}}_{G L} \cap \widetilde{\mathcal{B}}$. In view also of the results about the dynamical determinant (2.9) recalled below, it is legitimate to call the eigenvalue: $11 \Sigma_{B T}=\left\{\tilde{\rho}_{j}\right\}_{j=0}^{N}$ (repeated with multiplicity, ordered with decreasing modulus) of $\widetilde{\mathcal{L}}$ of modulus $\left|\tilde{\rho}_{j}\right|>\tilde{\rho}_{\text {ess }}$ the resonances of $F$. We cal12 the $N_{1} \leq N$ resonances $\left\{\tilde{\rho}_{j}\right\}_{j=1}^{N_{1}}$ with $1 \leq\left|\tilde{\rho}_{j}\right|<e^{h_{t o p}(F)}$ the deviation resonances of $F$.

Since $\sup _{\mu \in \operatorname{Erg}(F)}\left\{h_{\mu}-\tau \ell_{\mu}^{u}\right\}<0$ if $\tau>1$, it is not hard to see that $\tilde{\rho}_{B T}<1$ if

$$
r>r_{1}:=1+\epsilon+\sup _{\mu \in \operatorname{Erg}(F)} \frac{\ell_{\mu}^{u}-\ell_{\mu}^{s}}{-\ell_{\mu}^{s}}, .
$$

(If $F$ preserves area, (2.7) reduces to $r_{1}=3+\epsilon$, taking $t$ and $-s$ arbitrarily close to $(r-1) / 2$.) Note that (2.7) implies

$$
r_{1} \leq 1+\epsilon+\frac{-\log \Lambda_{s}+\log \Lambda_{u}}{-\log \lambda_{s}},
$$

with $\Lambda_{u} \geq \lambda_{u}$ and $\Lambda_{s} \leq \lambda_{s}$ the strongest expansion, respectively contraction of $F$.

2.4. The determinant $\tilde{d}(z)$ of $\widetilde{\mathcal{L}}$. Theorems 1.5 and $\S 2$ of [3] (see also [2, Chap. $6]$ ) give that the dynamical determinant

$$
d_{\tilde{F}, \tilde{g}}(z)=\exp -\sum_{n=1}^{\infty} \frac{z^{n}}{n} \sum_{(x, v) \in \text { Fix } \tilde{F}^{n}} \frac{\prod_{k=0}^{n-1} \tilde{g}\left(\tilde{F}^{k}(x, v)\right)}{\operatorname{det}\left(\operatorname{Id}-D \tilde{F}^{-n}(x, v)\right)}
$$

admits a holomorphic extension to the open disc of radius $\tilde{\rho}_{B T}^{-1}$, in which the zeroes of $d_{\tilde{F}, \tilde{g}}(z)$ are exactly the inverses of the eigenvalues of $\widetilde{\mathcal{L}}$ of modulus $>\tilde{\rho}_{B T}$ (the order of the zero coincides with the algebraic multiplicity of the eigenvalue).

2.5. Statement of results. We now state our main result, using the notation from $\$ 2.3$, in particular (2.3), (2.4), and (2.9):

Theorem 2.1 (Absence of deviation resonances). Fix $r>1$ and let $F$ be a $C^{r}$ Anosov diffeomorphism of the two-torus preserving the orientation of $E^{s}$.

The only zero of the dynamical determinant $d_{\tilde{F}, \tilde{g}}(z)$ in the closed disc of radius $\min \left\{1, \tilde{\rho}_{B T}^{-1}\right\}$ is a simple zero at $e^{-h_{t o p}(F)}$. In particular the spectrum of the operator $\widetilde{\mathcal{L}}$ (acting on $\widetilde{\mathcal{B}}$ ) outside of the open disc of radius $\max \left\{1, \tilde{\rho}_{B T}\right\}$ consists in a simple eigenvalue at $e^{h_{t o p}(F)}$. (In the notation of 2.3, we have $N_{1}=0$.)

Remark 2.2. If $F$ does not preserve the orientation of $E^{s}$, the same result holds up to introducing a non-mixing extension $\tilde{F}$ of $F$ such that $\tilde{F}^{-1}$ exchanges $\Omega_{+}^{s}$ and $\Omega_{-}^{s}$. The only difference is that there are two maximal eigenvalues, $\pm e^{h_{\text {top }}(F)}$.

Recalling $r_{0}$ from $\$ 1.1$ (see also Remark 1.1), and using the notation from $\$ 2.3$, in particular (2.6) and (2.7), we get:

\footnotetext{
${ }^{11}$ The eigenvalues $\left\{\tilde{\rho}_{j}\right\}_{j=1}^{N}$ are in bijection with the possible poles $v_{j}$ of the Fourier transform of the correlation function of the measure of maximal entropy of $F$, see also Corollary 2.5

${ }^{12}$ Note that $N_{1}=N_{1, G L}$.
} 
Corollary 2.3 (No deviations for horocycle ergodic integrals). Let $F: \mathbb{T}^{2} \rightarrow \mathbb{T}^{2}$ be a $C^{r}$ Anosov diffeomorphism which preserves the orientation of $E^{s}$. If $r \geq$ $\max \left\{r_{0}, r_{1}\right\}$, so that $\tilde{\rho}_{\text {ess }}<1$, then for any $\delta>0$ ther 13 exists $C^{\prime}<\infty$ such that for any $C^{r-1}$ function $f: \mathbb{T}^{2} \rightarrow \mathbb{C}$

$$
\sup _{x}\left|H_{x, T}(f)-T \cdot \mu^{s}(f)\right| \leq C^{\prime}\left(T^{\theta_{\text {min }}^{\prime}}\|f\|_{C^{r-1}}+\sup |f|\right), \forall T>0,
$$

where (taking small enough $\delta$ )

$$
\theta_{\text {min }}^{\prime}=\frac{\log \tilde{\rho}_{e s s}+\delta}{h_{\text {top }}}<0 \text { if } N=0, \theta_{\text {min }}^{\prime}=\frac{\delta+\log \max \left\{\tilde{\rho}_{j}\right\}_{j=1}^{N}}{h_{\text {top }}}<0 \text { if } N \geq 1 .
$$

In particular $\mu^{s}(f)=0$ if and only if $f$ is a continuous coboundary.

Remark 2.4. Our result does not exclude the existence of obstructions to Lipschitz (or Hölder) regularity of the solution $\bar{f}$ of the cohomological equation for $f$ when $\mu^{s}(f)=0$ [7, end of $\S 5.1 .2$, Remark 5.10]. Such constructions involve a different transfer operator (see the proof of [7, Theorem 2.12]).

Proof of Corollary 2.3. Since we assume $\tilde{\rho}_{\text {ess }}<1$, the corollary follows from the expansion (1.2) from [7, Theorem 2.8] combined with Theorem 2.1 and (1.3). (If $N \geq 1$, taking $\delta \in\left(0,-\log \max \left\{\tilde{\rho}_{j}\right\}_{j=1}^{N}\right)$ handles the possible Jordan blocks. This could be replaced by an appropriate power of $\log T$ in front of $T^{\theta_{\text {min }}^{\prime}}$.)

Our final result is about the unique measure of maximal entropy $\mu_{\text {top }}$ of $F$ :

Corollary 2.5 (Rates of mixing for the measure of maximal entropy). Let $r>1$ and let $F: \mathbb{T}^{2} \rightarrow \mathbb{T}^{2}$ be a $C^{r}$ Anosov diffeomorphism on the two-torus. If $\tilde{\rho}_{B T}<1$, then there exist $C<\infty$ and $\rho_{\text {top }}<e^{-h_{\text {top }}(F)}$ such that for any $C^{r-1}$ function 114 $f_{1}, f_{2}: \mathbb{T}^{2} \rightarrow \mathbb{C}$

$$
\left|\int\left(f_{1} \circ F^{k}\right) f_{2} d \mu_{t o p}-\int f_{1} d \mu_{t o p} \int f_{2} d \mu_{t o p}\right| \leq C \rho_{t o p}^{k}\left\|f_{1}\right\|_{C^{r-1}}\left\|f_{2}\right\|_{C^{r-1}}, \quad \forall k \geq 0 .
$$

If $\tilde{\rho}_{B T} \geq 1$, then for any $\rho_{\text {top }}>e^{-h_{\text {top }}(F)} \tilde{\rho}_{B T}$ there exists $C$ such that the above bound holds. (Note that $e^{-h_{\text {top }}(F)} \tilde{\rho}_{B T}<1$.)

Recalling (2.7), note that if $r \geq r_{1}$ then $\tilde{\rho}_{B T}<1$. Clearly, if $\tilde{\rho}_{B T} \geq 1$, then the bound $\rho_{t o p}$ given by Corollary 2.5 is $\geq e^{-h_{t o p}(F)}$.

Remark 2.6 (Comparing with the results of [6]). In our setting, Forni's results imply in particular 15 that if $F$ is $C^{r}$ for $r>2$, there exists $C<\infty$ such that for any $C^{1}$ functions $f_{1}$ and $f_{2}$, we have

$$
\left|\int\left(f_{1} \circ F^{k}\right) f_{2} d \mu_{t o p}-\int f_{1} d \mu_{t o p} \int f_{2} d \mu_{t o p}\right| \leq C k^{2} e^{-k h_{t o p}}\left\|f_{1}\right\|_{C^{1}}\left\|f_{2}\right\|_{C^{1}}, \forall k \geq 0 .
$$

It suffices to assume that $r>2$ and $f_{1}$ and $f_{2}$ are $C^{1}$ to get (2.11), but the error term there is not as good as in Corollary 2.5.

\footnotetext{
${ }^{13}\|f\|_{C^{r-1}}$ can be replaced by a weaker norm. In the area preserving case, $C^{(r-1) / 2}$.

${ }^{14}$ The $C^{r-1}$ norms can be replaced by weaker norms. In the area preserving case, $C^{(r-1) / 2}$.

${ }^{15}$ We do not discuss Forni's analog of Corollary 2.3 which contains an additional logarithmic factor $(\log T)^{2}$ in the error term.
} 
Proof of Corollary 2.5. We may assume that $F$ preserves the orientation of $E^{s}$, since otherwise we can replace $F$ by $F^{2}$ (the two maps have the same measure of maximal entropy and $h_{\text {top }}\left(F^{2}\right)=2 h_{\text {top }}(F)$ ).

Let $\mu^{u}=\pi^{*}\left(\tilde{\mu}^{u}\right)$ where $\widetilde{\mathcal{L}}\left(\tilde{\mu}^{u}\right)=e^{h_{\text {top }}(F)} \tilde{\mu}^{u}$, normalised by $\mu^{s}\left(\mu^{u}\right)=\tilde{\mu}^{s}\left(\tilde{\mu}^{u}\right)=1$. By [9], the distribution $\varphi \mapsto \mu^{s}\left(\varphi \mu^{u}\right)$ is in fact the unique measure of maximal entropy $\mu_{t o p}$ of $F$. (The fact that [9] use another Banach space does not matter by 3. App. A.2].) Then using the spectral decomposition for $\widetilde{\mathcal{L}}$ and the information from Theorem 2.1 gives the claim, just like in [9. (For example, if $\tilde{\rho}_{B T}<1$, we may choose $\rho_{\text {top }}$ as follows: If $\widetilde{\mathcal{L}}$ has no eigenvalue except $e^{h_{t o p}(F)}$ of modulus strictly larger than $\tilde{\rho}_{B T}$, then we can take any $\rho_{t o p}>\tilde{\rho}_{B T} / e^{h_{t o p}(F)}$ since $\tilde{\rho}_{B T}<1$. Otherwise, letting $\tilde{\rho}_{1}$ be the eigenvalue of $\widetilde{\mathcal{L}}$ of largest modulus $\neq e^{h_{t o p}(F)}$, we have $\left|\tilde{\rho}_{1}\right|<1$ by Theorem 2.1. and for any fixed integer $q \geq 0$, taking $\rho_{t o p}>\left|\tilde{\rho}_{1}\right| / e^{h_{t o p}(F)}$, we have $k^{q}\left|\tilde{\rho}_{1}\right|^{k} e^{-k h_{t o p}(F)}<C \rho_{\text {top }}^{k}$ for all $k$.)

\section{Proof of Theorem 2.1}

In order to prove Theorem 2.1, we introduce the following notation: If $\Phi: M \rightarrow$ $M$ is an Axiom A diffeomorphism with a basic set $X$ and $\phi: X \rightarrow \mathbb{C}$ admits a Hölder extension to a neighbourhood of $X$, we set $\phi^{(n)}=\prod_{k=0}^{n-1}\left(\phi \circ \Phi^{k}\right)$, and

$$
d_{\Phi, \phi}(z)=\exp -\sum_{n=1}^{\infty} \frac{z^{n}}{n} \sum_{y \in \operatorname{Fix} \Phi^{n} \cap X} \frac{\phi^{(n)}(y)}{\mid \operatorname{det}\left(\operatorname{Id}-D \Phi^{-n}(y)\right)} .
$$

For such a fixed $\Phi$ and $X$, we say that $\phi_{1}<_{P e r} \phi_{2}$ if $\phi_{1}, \phi_{2}: X \rightarrow \mathbb{R}_{*}^{+}$satisfy

$$
\limsup _{n \geq 1} \sup _{y \in \operatorname{Fix}^{n} \cap X} \frac{\left(\phi_{1}^{(n)}(y)\right)^{1 / n}}{\left(\phi_{2}^{(n)}(y)\right)^{1 / n}}<1 .
$$

Finally, the following two elementary facts will be used in the proofs: For a $2 \times 2$ matrix $Q$, we have $\operatorname{Det}(\operatorname{Id}-Q)=1+\operatorname{Det} Q-\operatorname{tr} Q$. If $\widetilde{Q}$ is a $3 \times 3$ matrix of the form $\widetilde{Q}=\left(\begin{array}{cc}Q & q \\ 0 & \eta\end{array}\right)$, with $q$ an arbitrary column vector in $\mathbb{R}^{2}$ and $\eta \in \mathbb{R}$, we have

$$
\operatorname{Det}(\operatorname{Id}-\widetilde{Q})=(1+\operatorname{Det} Q-\operatorname{tr} Q)(1-\eta) \text {. }
$$

If $F=A$ (so that $r=\infty$ ) and $\sigma_{A}=1$ then the above facts for $Q=A^{-n}$ give 16

$$
d_{\tilde{F}, \tilde{g}}(z)=\prod_{j=0}^{\infty}\left(1-z \lambda_{A}^{1-2 j}\right) .
$$

(We shall not use the above expression.)

Proof of Theorem 2.1. As a warmup, we assume that $r>3$, we fix $\epsilon>0$ small, and we consider the transfer operator

$$
\mathcal{L} \varphi(x)=\frac{\varphi\left(F^{-1}(x)\right)}{|\operatorname{Det} D F|_{E^{s}} \mid \circ F^{-1}(x)}
$$

acting on the Banach space of distributions $\mathcal{B}=C^{(1+\alpha) / 2,-(1+\alpha) / 2}\left(F, \mathbb{T}^{2}\right)$ defined in [3, §4], where its spectral radius is $e^{h_{t o p}(F)}$, while its essential spectral radius is

\footnotetext{
${ }^{16}$ Formula (3.1] recovers the eigenvalues of the operator associated to $A$ obtained in 7 , $\left.\$ 5.2\right]$.
} 
strictly smaller than (recalling $\lambda>1$ from (2.5))

$$
\rho_{\text {ess }, \alpha}:=\frac{e^{h_{t o p}(F)}}{\lambda^{(1+\alpha) / 2}},
$$

where the regularity 17 of the bundle $E^{s}$ is $C^{1+\alpha}$ for some $\alpha>0$. By mixing, $e^{h_{\text {top }}(F)}$ is simple and is the only eigenvalue of $\mathcal{L}$ of maximal modulus. Finally $[3$, Theorem 1.5] also implies that the dynamical determinant

$$
d_{F, g^{s}}(z)=\exp -\sum_{n=1}^{\infty} \frac{z^{n}}{n} \sum_{x \in \text { Fix } F^{n}} \frac{\left(g^{s}\right)^{(n)}(x)}{\left|\operatorname{det}\left(\operatorname{Id}-D F^{-n}(x)\right)\right|},
$$

where $g^{s}=\left(|\operatorname{Det} D F|_{E^{s}} \mid\right)^{-1}$, is holomorphic in the disc of radius $1 / \rho_{e s s, \alpha}$ where its zeroes are the inverses of the eigenvalues of $\mathcal{L}$.

We will show that

$$
\operatorname{spec}\left(\left.\mathcal{L}\right|_{\mathcal{B}}\right) \cap\left\{\max \left(\rho_{\text {ess }, \alpha}, 1\right)<|\rho| \leq e^{h_{\text {top }}(F)}\right\}=\left\{e^{h_{\text {top }}(F)}\right\},
$$

and if, in addition $\rho_{\text {ess }, \alpha}<1$ (since $\alpha$ is generically smaller than 1 , this is a very strong assumption, even for large $r$ ), then $\mathcal{L}: \mathcal{B} \rightarrow \mathcal{B}$ does not have eigenvalues outside the open unit disc except for $e^{h_{t o p}}$. The starting point is the fact that the Artin-Mazur zeta function of $F$ coincides with the zeta function (2.1) of its linear model:

$$
\zeta_{F}(z)=\exp \sum_{n=1}^{\infty} \frac{z^{n}}{n}\left(\sum_{x \in \operatorname{Fix} F^{n}} 1\right)=\zeta_{A}(z) .
$$

Next, we use that for all $n$ and any $x \in \operatorname{Fix} F^{n}$,

$$
\begin{aligned}
1 & =\frac{\left|\operatorname{Det}\left(\operatorname{Id}-D F^{-n}(x)\right)\right|}{\left|\operatorname{Det}\left(\operatorname{Id}-D F^{-n}(x)\right)\right|}=\frac{\left|1+\operatorname{Det} D F^{-n}(x)-\operatorname{tr}\left(D F^{-n}(x)\right)\right|}{\left|\operatorname{Det}\left(\operatorname{Id}-D F^{-n}(x)\right)\right|} \\
& =\frac{\left(\sigma_{A}\right)^{n}\left(-1-\operatorname{Det} D F^{-n}(x)+\operatorname{tr}\left(D F^{-n}(x)\right)\right)}{\left|\operatorname{Det}\left(\operatorname{Id}-D F^{-n}(x)\right)\right|} .
\end{aligned}
$$

Since for any $x \in \operatorname{Fix} F^{n}$ we have (using orientation preserving on $E^{s}$ )

$$
\operatorname{tr}\left(D F^{-n}(x)\right)=\left(\left|\operatorname{Det} D F^{n}\right|_{E^{s}}(x) \mid\right)^{-1}+\left(\left.\operatorname{Det} D F^{n}\right|_{E^{u}}(x)\right)^{-1},
$$

setting $w=\sigma_{A} \cdot z$, it follows that

$$
\zeta_{A}(z)=\frac{d_{F, 1}(w) \cdot d_{F, 1 / \operatorname{Det} D F}(w)}{d_{F, g^{u}}(w) \cdot d_{F, g^{s}}(w)}
$$

where $g^{u}=\left(\left.\operatorname{Det} D F\right|_{E^{u}}\right)^{-1}$. Let $\beta>0$ b 18 such that $E^{u}(F)$ (and thus $g^{u}$ ) is $C^{1+\beta}$ and assume that $\epsilon<\beta<2$. Then there exists [3, Theorem 1.1] a Banach space $\mathcal{B}_{\beta}=$ $C^{(\beta-\epsilon) / 2,-(\beta-\epsilon) / 2}\left(F, \mathbb{T}^{2}\right)$ such that the transfer operator $\mathcal{L}_{F, g^{u}}(\varphi)=\left(g^{u} \varphi\right) \circ F^{-1}$ acting on $\mathcal{B}_{\beta}$ has spectral radius $<1$ (because $\left|g^{u}\right|<_{\text {Per }}|\operatorname{det} D F|^{-1}$ ) and essential spectral radius strictly smaller than its spectral radius, and, in addition, $d_{F, g^{u}}(w)$ is holomorphi 19 in a disc of radius $>1$.

We will see below that the two determinants in the numerator of (3.4) are holomorphic in the disc of radius $\rho_{S R B, \text { ess }}^{-1}>\max \left\{\rho_{\text {ess }, \alpha}^{-1}, 1\right\}$. Since $d_{F, g^{s}}(w)$ is the dynamical determinant of $\mathcal{L}$ which is holomorphic in the disc of radius $1 / \rho_{\text {ess }, \alpha}$,

\footnotetext{
${ }^{17}$ Of course, $\alpha=\infty$ in the linear case $F=A$, but Anosov proved that, generically, $\alpha<1$.

${ }^{18}$ In fact, $\beta=\alpha$ in the present setting.

${ }^{19}$ We also have that $d_{F, g^{u}}(w)$ cannot vanish inside a disc of radius $>1$. Since this determinant appears in the denominator, its zeroes do not matter to us.
} 
the domain 20 of holomorphy (and of spectral interpretation of zeroes) of the four determinants in the right hand side of (3.4) include the disc of radius $1 / \rho_{\text {ess }, \alpha}$.

Applying [3, Theorem 1.1] again, the transfer operator $\mathcal{L}_{F, S R B}(\varphi)=\frac{\varphi \circ F^{-1}}{|\operatorname{Det} D F| \circ F^{-1}}$ acting on $\mathcal{B}$ has essential spectral radius bounded by $\rho_{S R B, \text { ess }}<\rho_{\text {ess }, \alpha}$ (we have $\rho_{S R B, e s s} \leq \lambda^{-(r-1) / 2}$ ) and spectral radius one, with a simple eigenvalue at 1 as only eigenvalue on the unit circle. Therefore, if $\sigma_{A}=+1$ then $d_{F, 1 / \operatorname{Det} D F}(w)=$ $d_{F, 1 /|\operatorname{Det} D F|}(z)$ is holomorphic in the disc of radius $\rho_{S R B, e s s}^{-1}$, with $d_{F, 1 /|\operatorname{Det} D F|}(1)=$ 0 , and this determinant has no other zeroes in the closed unit disc. If $\sigma_{A}=-1$ then $d_{F, 1 / \operatorname{Det} D F}(w)=d_{F, 1 / \operatorname{Det} D F}(-z)$ is holomorphic in the disc of radius $\rho_{S R B, e s s}^{-1}$, with $d_{F, 1 /|\operatorname{Det} D F|}(-1)=0$, and this determinant has no other zeroes in the closed unit disc.

Finally, we claim that $d_{F, 1}(w)$ is holomorphic in the disc of radius $\rho_{S R B^{*} \text {,ess }}^{-1}>1$, with $d_{F, 1}(1)=0$, where 1 is simple zero and the only zero of $d_{F, 1}(w)$ in the closed unit disc: Indeed, $d_{F, 1}(w)$ can be viewed as the determinant of the operator

$$
\mathcal{L}_{F^{-1}, S R B} \varphi=|\operatorname{Det} D F| \cdot(\varphi \circ F)
$$

acting on the Banach space $\hat{\mathcal{B}}=C^{(t, s}\left(F^{-1}\right)\left(F^{-1}, \mathbb{T}^{2}\right)$, for suitable $t-(r-1)<$ $s<0$, associated to $F^{-1}$, on which its essential spectral radius is bounded by $\rho_{S R B^{*}, \text { ess }}<\tilde{\rho}_{\text {ess }, \alpha}$. The spectral radius of $\mathcal{L}_{F^{-1}, S R B}$ is equal to 1 , and, since $F^{-1}$ is mixing, the eigenvalue 1 is simple and it is the only eigenvalue of modulus equal to one. (The respective simple zeroes of $d_{F, 1}(z)$ and $d_{F, 1 /|\operatorname{Det} D F|}$ at $z=1$ account for the double zero of $\zeta_{F}(z)$ at $z=1$ when $\sigma_{A}=+1$. Otherwise, the simple zeroes at $z= \pm 1$ account for the zeroes of $\left(1-z^{2}\right)$.)

Assume by contradiction that $\mathcal{L}$ has an eigenvalue $\rho \neq e^{h_{\text {top }}(F)}$ with $|\rho| \geq 1$, then $d_{F, g}(1 / \rho)=0$. This would imply that $d_{F, 1}(1 / \rho)=0$ or $d_{F, 1 /|\operatorname{Det} D F|}(1 / \rho)=0$ (to get a cancellation), and both claims are impossible.

We now prove the theorem: The only differences are that we will need $3 \times 3$ matrices instead of $2 \times 2$ matrices, and six determinants instead of four. Also we only need that $E^{u}$ and $E^{s}$ are $C^{\gamma}$ for some $\gamma>0$. To fix ideas, assume first that $\tilde{\rho}_{B T}<1$ so that $\min \left\{1, \tilde{\rho}_{B T}^{-1}\right\}=1$. First

$$
\zeta_{\tilde{F}}(z)=\exp \sum_{n=1}^{\infty} \frac{z^{n}}{n}\left(\sum_{x \in \operatorname{Fix} \tilde{F}^{n}} 1\right)=\zeta_{A}(z) .
$$

(Recall (3.3) and (2.1).) Second, at any $\tilde{x}=(x, v)=\left(x, v_{1,+}^{s}(x)\right) \in$ Fix $\tilde{F}^{n}$, we have

$$
D \tilde{F}_{\tilde{x}}^{n}=\left(\begin{array}{cc}
D F_{x}^{n} & a \\
0 & \tilde{\lambda}^{n}(x)
\end{array}\right) \text { with } \tilde{\lambda}^{n}(x)=\frac{\left.\operatorname{Det} D F^{n}\right|_{E^{u}}(x)}{\left|\operatorname{Det} D F^{n}\right|_{E^{s}}(x) \mid},
$$

where $\left|\tilde{\lambda}^{n}(x)\right| \geq \frac{\lambda_{u}^{n}}{\lambda_{s}^{n}}$. Therefore, we may use for such $\tilde{x}$ the decomposition

$$
\begin{aligned}
1 & =\frac{\left|\operatorname{Det}\left(\operatorname{Id}-D \tilde{F}^{-n}(\tilde{x})\right)\right|}{\left|\operatorname{Det}\left(\operatorname{Id}-\tilde{D} F^{-n}(\tilde{x})\right)\right|} \\
& =\frac{\left(\left|1+\operatorname{Det} D F^{-n}(x)-\operatorname{tr}\left(D F^{-n}(x)\right)\right|\right)\left(1-\frac{\left|\operatorname{Det} D F^{n}\right|_{E^{s}(x) \mid}}{\left.\operatorname{Det} D F^{n}\right|_{E^{u}}(x)}\right)}{\left|\operatorname{Det}\left(\operatorname{Id}-D \tilde{F}^{-n}(\tilde{x})\right)\right|} .
\end{aligned}
$$

\footnotetext{
${ }^{20}$ Note that $d_{F, g^{u}}(z)$ is holomorphic in a disc of radius $>1$ with no zeroes in the closed unit disc even if $\rho_{\text {ess }, \alpha}>1$. The argument for $\widetilde{\mathcal{L}}$ will exploit a similar feature.
} 
Third, since for all $n$ and all $x \in \operatorname{Fix} F^{n}$,

$$
\begin{aligned}
& \left(\left|1+\operatorname{Det} D F^{-n}(x)-\operatorname{tr}\left(D F^{-n}(x)\right)\right|\right. \\
& \quad=\left(\sigma_{A}\right)^{n}\left(-1-\operatorname{Det} D F^{-n}(x)+\left(\left|\operatorname{Det} D F^{n}\right|_{E^{s}}(x) \mid\right)^{-1}+\left(\left.\operatorname{Det} D F^{n}\right|_{E^{u}}(x)\right)^{-1}\right),
\end{aligned}
$$

setting $w=\sigma_{A} \cdot z$, it follows that

$$
\begin{aligned}
\zeta_{A}(z) & =\frac{d_{\tilde{F}, 1}(w) \cdot d_{\tilde{F}, 1 / \operatorname{Det} D F}(w) \cdot d_{\tilde{F}, \tilde{g}^{u}}(w) \cdot d_{\tilde{F},\left(\tilde{g}^{u}\right)^{2} / g^{s}}(w)}{d_{\tilde{F}, g^{s}}(w) \cdot d_{\tilde{F}, \tilde{g}^{u}}(w) \cdot d_{\tilde{F}, \tilde{g}^{u} / \tilde{g}}(w) \cdot d_{\tilde{F},\left(\tilde{g}^{u}\right)^{2}}(w)} \\
& =\frac{d_{\tilde{F}, 1}(w) \cdot d_{\tilde{F}, 1, \operatorname{Det} D F}(w) \cdot d_{\tilde{F},\left(\tilde{g}^{u}\right)^{2} / \tilde{g}}(w)}{d_{\tilde{F}, \tilde{g}}(w) \cdot d_{\tilde{F}, \tilde{g}^{u} / \tilde{g}}(w) \cdot d_{\tilde{F},\left(\tilde{g}^{u}\right)^{2}}(w)},
\end{aligned}
$$

where $\tilde{g}$ was defined 21 in (2.2) and $\tilde{g}^{u}(x, v):=g^{u}(x)$ is $C^{\gamma}$.

As mentioned above, $d_{\tilde{F}, \tilde{g}}(z)$ is the dynamical determinant of $\widetilde{\mathcal{L}}$ acting on $\widetilde{\mathcal{B}}$, which is holomorphic in the disc of radius $1 / \tilde{\rho}_{B T}$.

It is easy to see that $d_{\tilde{F}, 1}(w)$ is the dynamical determinant of the transfer operator associated to the SRB measure of the mixing attractor $\tilde{F}^{-1}$ acting on the Banach space $\widetilde{\mathcal{B}}_{\tilde{F}^{-1}}=\mathcal{C}^{t, s}\left(\tilde{F}^{-1}, \mathcal{E}\right)$ for suitable $t-(r-1)<s<0$, associated to $\tilde{F}^{-1}$, so, by [3, Theorem 1.5 and $\left.\S 2\right]$, this factor is holomorphic in a disc of radius $\tilde{\rho}_{S R B^{*}, \text { ess }}^{-1}>\tilde{\rho}_{B T}^{-1}>1$, and its only zero in the closed unit disc is a simple zero at $w=1$. (If $\sigma_{A}=-1$ then this gives a zero at $z=-1$.)

Next, $d_{\tilde{F}, 1 /|\operatorname{Det} D F|}(w)$ is the dynamical determinant of the transfer operator $\mathcal{M}$ of the mixing repellor $\tilde{F}$ weighted by $1 /|\operatorname{Det} D F|$, acting on the Banach space $\widetilde{\mathcal{B}}$. By the Pesin entropy formula the pressure 22 of $-\log |\operatorname{Det} D F|_{E^{u}} \mid$ for the attractor $F$ is equal to zero. Since this pressure coincides with the pressure of $-\log |\operatorname{Det} D F|_{E^{u}} \mid$ for the repellor $\tilde{F}$, applying [3, Theorem 1.5] the determinant $d_{\tilde{F}, 1 /|\operatorname{Det} D F|}(w)$ is holomorphic in a disc of radius $\tilde{\rho}_{S R B, e s s}^{-1}>\tilde{\rho}_{B T}^{-1}>1$ and its only zero in the closed unit disc is a simple zero at $w=1$. (The fixed point of $\mathcal{M}$ is the SRB measure of $F$ in $x$ multiplied by a Dirac mass at $v_{x,+}^{1}$ ) If $\sigma_{A}=-1$, replacing $\mathcal{M}$ by $-\mathcal{M}$, we get that the determinant $d_{\tilde{F}, 1 / \operatorname{Det} D F}(w)$ is holomorphic in a disc of radius $\tilde{\rho}_{S R B, e s s}^{-1}>\tilde{\rho}_{B T}^{-1}>1$ and its only zero in the closed unit disc is a simple zero at $w=-1$, giving a zero at $z=1$.

The last determinant in the numerator of (3.5) is $d_{\tilde{F},\left(\tilde{g}^{u}\right)^{2} / \tilde{g}}(w)$, which is associated to a transfer operator $\mathcal{N}$ of spectral radius $<1$ on the Banach space $\widetilde{\mathcal{B}}_{\gamma}=C^{\gamma / 2,-\gamma / 2}(\tilde{F}, \mathcal{E})$ using $\left(\tilde{g}^{u}\right)^{2} / \tilde{g}(z)<_{\text {Per }}|\operatorname{det} D F|^{-1}$ (the essential spectral radius of $\mathcal{N}$ is $<1$, but we do not claim that this essential spectral radius is smaller than $\left.\tilde{\rho}_{B T}\right)$. Therefore, $d_{\tilde{F},\left(\tilde{g}^{u}\right)^{2} / \tilde{g}}(w)$ cannot vanish on the closed unit disc.

Finally, the spectral radii of the two remaining operators in the denominator of (3.5) acting on $\widetilde{\mathcal{B}}_{\gamma}$ are smaller than 1 since

$$
\left|\tilde{g}^{u}\right| / \tilde{g}<_{\text {Per }}|\operatorname{det} D F|^{-1}, \quad\left(\tilde{g}^{u}\right)^{2}<_{\text {Per }}|\operatorname{det} D F|^{-1} .
$$

So the right-hand-side of (3.5) is a quotient of two holomorphic functions in a disc of radius $>1$, such that the only zeroes of the numerator in the closed unit disc are a double zero at $z=1$ when $\sigma_{A}=1$, and simple zeroes at $z= \pm 1$ otherwise.

\footnotetext{
${ }^{21}$ Note that $\tilde{g}\left(x, v_{1,+}^{s}(x)\right)=g^{s}(x)$.

${ }^{22}$ The pressure of $-\log |\operatorname{Det} D \tilde{F}|_{E^{u}} \mid$ for the repellor $\tilde{F}$ is strictly smaller than zero.
} 
It follows that the only possible zero of $d_{\tilde{F}, \tilde{g}}(z)$ in the closed unit disc is a simple zero at $\lambda_{A}^{-1}=e^{-h_{t o p}(F)}<1$. This ends the proof of the theorem if $\tilde{\rho}_{B T}<1$.

If $\tilde{\rho}_{B T} \geq 1$, the same argument works, replacing the unit disc by the disc of radius $\tilde{\rho}_{B T}$ for spectral claims and by the disc of radius $\tilde{\rho}_{B T}^{-1}$ for determinants.

Remark 3.1 (Higher dimension). Consider an Anosov diffeomorphism $F$ with $d_{s}=1$ and $d_{u}=2$. Then, assuming that $D F$ preserves the orientation of $E^{s}$, and that the eigenvalues of the hyperbolic linear matrix $A$ conjugated to $F$ satisfy $\lambda_{s}<1<$ $\lambda_{u, \min }<\lambda_{u, \max }$, the unweighted Artin-Mazur zeta function of $F$ is (note that $\lambda_{s} \lambda_{u, \min } \lambda_{u, \max }=1$, and, since $d$ is odd, +1 and $(-1)^{d} \operatorname{det}\left(A^{-n}\right)$ cancel for all $n$ )

$$
\zeta_{A}(z)=\frac{\left(1-\lambda_{s} z\right)\left(1-\lambda_{u, \min } z\right)\left(1-\lambda_{u, \max } z\right)}{\left(1-z / \lambda_{s}\right)\left(1-z / \lambda_{u, \min }\right)\left(1-z / \lambda_{u, \max }\right)} .
$$

Factoring the zeta function as a product of dynamical determinants for an extended dynamics $\tilde{F}$ as in the proof of Theorem 2.1, one cannot exclude a priori eigenvalues of modulus $\geq 1$ for the operator (in the denominator) weighted by $1 /|\operatorname{det} D F|_{E^{s}} \mid$, since the corresponding zeroes could be cancelled by the determinants of the transfer operators in the numerator weighted by $\left.\operatorname{det} D F\right|_{E_{m a x}^{u}}$ or even $\left.\operatorname{det} D F\right|_{E_{m i n}^{u}}$, which also have spectral radius strictly larger than 1.

\section{Appendix A. Properties of Giulietti-Liverani flows}

Let $h^{t}$ be a (not necessarily unit speed) Giulietti-Liverani flow of a $C^{r}$ Anosov diffeomorphism $F$. If $r>2$, since the stable bundle of $F$ is $C^{1+\alpha}$ for some $\alpha>0$ (see e.g. 11] and references therein), the map $x \mapsto h^{t}(x)$ is $C^{1+\alpha}$. Clearly, $h^{t}$ cannot have fixed points or periodic orbits. It is well known that a periodic orbit-free flow on $\mathbb{T}^{2}$ has a global transversal, with the corresponding Poincaré map topologically conjugated to an irrational rigid rotation $R_{\omega}$, and that the flow is uniquely ergodic (and thus minimal, using the conjugacy with the linear model). By [7, Lemma 1.1], the rotation number $\omega$ of the rotation thus associated to a Giulietti-Liverani flow satisfie 23

$$
b \omega^{2}+(a-d) \omega-c=0,
$$

where $A=\left(\begin{array}{ll}a & b \\ c & d\end{array}\right) \in \operatorname{PSL}(2, \mathbb{Z})$ is the hyperbolic linear toral automorphism topologically conjugated to $F$. In particular, $b \neq 0$, and the continued fraction of $\omega$ is periodic and thus of constant type. (The orientation preserving assumption also implies that the contracting eigenvalue of $A$ is positive.)

Finally, by [7, Lemma 1.1], if $r \geq 3$, any $C^{r}$ periodic orbit-free flow $h_{0}^{t}$ on $\mathbb{T}^{2}$ whose Poincaré map $P_{0}$ has rotation number $\omega$ satisfying (A.1) for some hyperbolic matrix $A \in\left(\begin{array}{ll}a & b \\ c & d\end{array}\right) \in P S L(2, \mathbb{Z})$, with positive contracting eigenvalue, is the (non necessarily unit speed) Giulietti-Liverani flow of an Anosov diffeomorphism $F_{0}$, which is $C^{r^{\prime}}$ for all $r^{\prime}<r-1$ : Indeed, the $C^{r}$ circle diffeomorphism $P_{0}$ has rotation number $\omega$ of constant type and is thus conjugated with the rigid rotation $R_{\omega}$ via a $C^{r^{\prime}}$ circle diffeomorphism $\phi$ [12, Théorème fondamental, p. 9], for any $r^{\prime}<r-1$. Let $h_{1}^{t}$ be the unit speed $C^{r}$ reparametrisation of $h_{0}^{t}$. Then the (unit speed) linear flow $h_{\omega}^{t}$ over $R_{\omega}$ is the Giulietti-Liverani flow of the hyperbolic automorphism $A$.

\footnotetext{
${ }^{23}$ As pointed out by the Editor, in the first statement of 7 Lemma 1.1] the sentence "it is topologically conjugated to a rigid rotation with rotation number $\omega$ such that..." should be replaced by "it is topologically orbit equivalent with a flow whose Poincaré map on a global transversal has rotation number $\omega$ such that..."
} 
In addition, the flow $h_{\omega}^{t}$ is conjugated with $h_{1}^{t}$ via a $C^{r^{\prime}}$ toral diffeomorphism $\Phi$ which coincides with $\phi$ on the transversal and maps the stable lines of $h_{\omega}^{t}$ to the orbits of $h_{i}^{t}, i=0,1$. Finally, $h_{1}^{t}$ is the (unit speed) Giulietti-Liverani flow of the $C^{r^{\prime}}$ Anosov diffeomorphism $F_{0}=\Phi \circ A \circ \Phi^{-1}$. (Use that the stable manifolds of $F_{0}$ coincide with the orbits of $h_{i}^{t}, i=0,1$.)

\section{REFERENCES}

[1] A. Adam, Horocycle averages on closed manifolds and transfer operators, arXiv:1809.04062 (2018)

[2] V. Baladi Dynamical Zeta Functions and Dynamical Determinants for Hyperbolic Maps, Ergebnisse der Mathematik und ihrer Grenzgebiete, 68 Springer, Cham (2018)

[3] V. Baladi and M. Tsujii, Dynamical determinants and spectrum for hyperbolic diffeomorphisms, in Geometric and Probabilistic Structures in Dynamics, 29-68, Contemp. Math. 469, Amer. Math. Soc. (2008)

[4] J. Carrand, Logarithmic bounds for ergodic averages of constant type rotation number flows on the torus: a short proof, arXiv:2012.07481 (2020)

[5] L. Flaminio and G. Forni, Invariant distributions and time averages for horocycle flows, Duke Math. J. 119 465-526 (2003)

[6] G. Forni, On the equidistribution of unstable curves for pseudo-Anosov diffeomorphisms of compact surfaces, arXiv:2007.03144

[7] P. Giulietti and C. Liverani, Parabolic dynamics and anisotropic Banach spaces, JEMS 21 2793-2858 (2019)

[8] S. Gouëzel and C. Liverani, Banach spaces adapted to Anosov systems, Ergodic Theory Dynam. Systems 26 189-217 (2006)

[9] S. Gouëzel and C. Liverani, Compact locally maximal hyperbolic sets for smooth maps: fine statistical properties, J. Diff. Geom. 79 (2008)

[10] A. Katok and B. Hasselblatt, Introduction to the Modern Theory of Dynamical Systems, Encyclopedia Math. and Applications 54 Cambridge University Press (1995)

[11] B. Hasselblatt, Regularity of the Anosov splitting and of horospheric foliations, Ergodic Theory Dynam. Systems 14 645-666 (1994)

[12] M.-R. Herman, Sur la conjugaison différentiable des difféomorphismes du cercle à des rotations, Inst. Hautes Études Sci. Publ. Math. 49 (1979) 5-233.

[13] K. Hiraide, A simple proof of the Franks-Newhouse theorem on codimension-one Anosov diffeomorphisms, Ergod. Th. Dynam. Sys. 21 801-806 (2001)

[14] R. McCutcheon, The Gottschalk-Hedlund theorem, Amer. Math. Monthly 106 670-672 (1999)

Laboratoire de Probabilités, Statistique et Modélisation (LPSM), CNRS, Sorbonne Université, Université de Paris, 4, Place Jussieu, 75005 Paris, France

Email address: baladi@lpsm.paris 